\title{
Les délaissés urbains : supports de nouvelles pratiques et représentations de la nature spontanée? Comparaison des représentations des gestionnaires et des habitants Urban Wastelands: Can They Support New Uses and Perceptions of Spontaneous Nature? Comparison of Residents and Planners' Perceptions
}

\author{
Marion Brun, Francesca Di Pietro et Denis Martouzet
}

Volume 14, numéro 2, mai 2019

Sur le thème : Nature et action

URI : https://id.erudit.org/iderudit/1062509ar

DOI : https://doi.org/10.7202/1062509ar

\section{Aller au sommaire du numéro}

\section{Éditeur(s)}

Prise de parole

\section{ISSN}

1712-8307 (imprimé)

1918-7475 (numérique)

\section{Découvrir la revue}

Citer cet article

Brun, M., Di Pietro, F. \& Martouzet, D. (2019). Les délaissés urbains : supports de nouvelles pratiques et représentations de la nature spontanée?

Comparaison des représentations des gestionnaires et des habitants. Nouvelles perspectives en sciences sociales, 14(2), 153-184.

https://doi.org/10.7202/1062509ar

\section{Résumé de l'article}

Les délaissés urbains sont des espaces sans fonction officielle et en rupture avec le tissu urbain environnant. Espaces transitoires présentant souvent une végétation spontanée, les délaissés constituent un habitat pour de nombreuses espèces végétales et leur intérêt pour la biodiversité urbaine est maintenant reconnu. Ils représentent aussi des lieux privilégiés pour augmenter le contact des habitants avec la nature et répondre à la croissante demande sociale de nature en ville. Par ailleurs, l'intérêt des délaissés urbains comme objet d'aménagement est largement documenté en urbanisme; toutefois les représentations des urbanistes et gestionnaires sur les délaissés ont été peu étudiées. L'objectif de ce travail est d'explorer les représentations comparées des délaissés urbains par les gestionnaires (propriétaires et non propriétaires de délaissés) et les habitants riverains de ces espaces. Une méthodologie spécifique d'enquête pour chaque type d'acteur a été mise en place sur nos sites d'étude, les agglomérations de Tours et Blois. Une partie des habitants riverains adoptent sur les délaissés des pratiques temporaires, qui sont souvent tolérées par les propriétaires et n'affectent pas les usages définitifs du terrain, qui sont principalement la construction et la commercialisation des délaissés, lorsque les terrains sont constructibles. Malgré des points de vue contrastés entre gestionnaires et habitants, les délaissés sont majoritairement représentés comme des espaces non entretenus, abandonnés, végétalisés et temporaires. Toutefois pour certains habitants ce devenir incertain revêt un aspect positif, car il est associé à une idée de « liberté » qui confirme la demande sociale d'espaces de respiration dans la ville de la part de certains habitants. Deux pistes pour une meilleure intégration des délaissés dans la ville sont formulées en conclusion, qui permettraient de valoriser pour les habitants, mais aussi pour les gestionnaires, ces espaces urbains en attente. 


\section{Les délaissés urbains : supports de nouvelles pratiques et représentations de la nature spontanée? Comparaison des représentations des gestionnaires et des habitants}

\section{Marion Brun, Francesca Di Pietro et \\ Denis MARTOUZET Université de Tours, UMR CITERES}

es délaissés urbains, ces espaces "indécis, dépourvus de comme l'énonce Gilles Clément ${ }^{1}$, sont des espaces sans fonction officielle et donc en rupture avec le tissu urbain environnant. Espaces transitoires présentant souvent une végétation spontanée, les délaissés constituent un habitat pour de nombreuses espèces végétales et leur intérêt pour la biodiversité urbaine a été mis en évidence dans une synthèse bibliographique ${ }^{2}$. Les potentialités écologiques sont toutefois ambivalentes et peuvent s'accompagner

Gilles Clément, Manifeste du tiers paysage, Montreuil, Sujet/Objet éditions, 2007, p. 12.

2 Sébastien Bonthoux et al., " How Can Wastelands Promote Biodiversity in Cities? A Review ", Landscape and Urban Planning, $\mathrm{n}^{\circ}$ 132, 2014, p. 79-88. 
de risques pour la santé humaine ${ }^{3}$ et le bien-être ${ }^{4}$, notamment dans les villes marquées par la désindustrialisation ${ }^{5}$. Dans le cadre d'un projet de recherche portant sur les délaissés urbains et les espèces envahissantes (projet régional DUE), dans deux agglomérations de taille moyenne (Tours et Blois), l'étude de 179 délaissés a confirmé leur intérêt pour la conservation de la biodiversité urbaine ${ }^{6}$.

En outre les délaissés urbains, disséminés à de multiples endroits dans la ville et présents dans des villes de toutes tailles, représentent des lieux privilégiés pour augmenter le contact des habitants avec la nature. Ils pourraient alors offrir des espaces de nature de proximité ${ }^{7}$ et répondre à la croissante demande sociale de nature en ville ${ }^{8}$. Une synthèse bibliographique recense à ce propos la multiplicité de bénéfices apportés aux habitants par les

$3 \quad$ Clare Bambra et al., "Healthy Land? An Examination of the Area-Level Association between Brownfield Land and Morbidity and Mortality in England ", Environment and Planning A: Economy and Space, vol. 46, n 2, 2014, p. 433-454; Clare Bambra et al., "This Divided Land: An Examination of Regional Inequalities in Exposure to Brownfield Land and the Association with Morbidity and Mortality in England ", Health and Place, $\mathrm{n}^{\circ}$ 34, 2015, 257-69; Jill Litt et Thomas A. Burke, «Uncovering the Historic Environmental Hazards of Urban Brownfields ", Journal of Urban Health, vol. 79, n 4, 2002, p. 464-81.

4 Christian Krekel, Jens Kolbe et Henry Wüstemann, «The Greener, The Happier? The Effects of Urban Green and Abandoned Areas on Residential Well-Being ", Ecological Economics, n 121, 2016, p. 117-27.

5 Christopher B. Riley et al., "Asset or Liability? Ecological and Sociological Tradeoffs of Urban Spontaneous Vegetation on Vacant Land in Shrinking Cities ", Sustainability, vol. 10, n 7, 2018, p. 1-19.

6 Marion Brun et al., "Les services de support de diversité floristique rendus par les délaissés urbains ", Environnement Urbain / Urban Environment, n ${ }^{\circ} 11$, 2017, https://journals.openedition.org/eue/1500, consulté le consulté le 12 juin 2012.

7 Christoph D. D. Rupprecht et Jason A. Byrne, "Informal Urban GreenSpace: Comparison of Quantity and Characteristics in Brisbane, Australia and Sapporo, Japan ", PLoS ONE, vol. 9, n 6, 2014, https://journals.plos. org/plosone/article?id=10.1371/journal.pone.0099784, consulté le 12 juin 2017; Christoph D. D. Rupprecht et al., " "It's Real, Not Fake Like a Park": Residents' Perception and Use of Informal Urban Green-Space in Brisbane, Australia and Sapporo, Japan ", Landscape and Urban Planning, ${ }^{\circ}$ 143, 2015, p. 205-218.

8 Emmanuel Boutefeu, La demande sociale de nature en ville, enquête auprès des habitants de l'agglomération lyonnaise, Paris, PUCA/CERTU, 2005. 
espaces verts informels ${ }^{9}$. Toutefois la végétation des délaissés, parfois perçue comme désordonnée, peut être vue par les habitants comme un élément dégradant de l'environnement ${ }^{10}$. Les citadins souffriraient d'une "perte d'expérience de la nature ${ }^{11}$ ", qui les amènerait à ne plus apprécier les espaces de nature lorsqu'ils ne sont pas transformés ${ }^{12}$. Certains délaissés demeurent ainsi peu fréquentés, parfois rejetés par les résidents proches côtoyant le délaissé pendant son temps de veille ${ }^{13}$. Ces différences d'appréciation des délaissés urbains et de leur végétation spontanée sont confirmées par d'autres études ${ }^{14}$. Elles peuvent être liées aux représentations des espaces de nature, influencées de façon ambivalente par le modèle émergent du "sauvage ${ }^{15}$ ", mais aussi à la densité d'espaces semi-naturels, plus élevée en zone péri-urbaine qu'au centre-ville ${ }^{16}$, les centres urbains souffrant d'une offre de nature insuffisante ${ }^{17}$. De façon générale, les travaux sur les usages des délaissés urbains par les habitants se développent depuis

$\overline{9}$ Christoph D. D. Rupprecht et Jason A. Byrne, «Informal Urban Greenspace: A Typology and Trilingual Systematic Review of Its Role for Urban Residents and Trends in the Literature ", Urban Forestry and Urban Greening, vol. 13, $\mathrm{n}^{\circ}$ 4, 2014, p. 597-611.

10 Christoph D. D. Rupprecht et al., " "It's Real, Not Fake Like a Park”... ", op. cit.

11 Assaf Schwartz et al., "Outstanding Challenges for Urban Conservation Research and Action ", Global Environmental Change, vol. 28, ${ }^{\circ} 1,2014$, p. 39-49.

12 Paul H. Gobster et Lynne M. Westphal, "The Human Dimensions of Urban Greenways: Planning for Recreation and Related Experiences ", Landscape and Urban Planning, vol. 68, $\mathrm{n}^{\text {os }}$ 2-3, 2004, p. 147-165: James R. Miller et Richard J. Hobbs, "Conservation Where People Live and Work ", Conservation Biology, vol. 16, $\mathrm{n}^{\circ}$ 2, 2002, p. 330-337.

13 Lauren Andres, "Les usages temporaires des friches urbaines, enjeux pour l'aménagement ", 2011, https://www.metropolitiques.eu/Les-usagestemporaires-des-friches.html, site consulté le 2 septembre 2018.

14 Julian Mathey et al., "Public Perception of Spontaneous Vegetation on Brownfields in Urban Areas-Results from Surveys in Dresden and Leipzig (Germany)", Urban Forestry and Urban Greening, n' 29, 2018, p. 384-392.

15 Bernadette Lizet, "Du terrain vague à la friche paysagée », Ethnologie française, $\mathrm{n}^{\circ}$ 4, 2010, p. 597-608.

16 Marie-Jo Menozzi, "Mauvaises herbes", qualité de l'eau et entretien des espaces ", Natures, sciences, sociétés, vol. 15, nº 2, 2007, p. 144-153.

17 Bernard Reygrobellet, La nature dans la ville. Biodiversité et urbanisme, Paris, La Documentation française, 2007. 
quelques années, en mettant l'accent sur les usages informels ${ }^{18}$, sur les processus d'appropriation de ces espaces ${ }^{19}$ et sur leurs représentations $s^{20}$.

Par ailleurs, l'intérêt des délaissés urbains comme objet d'aménagement est largement documenté en urbanisme, pour affirmer leur urbanité ambiguë ${ }^{21}$ ou leur potentiel pour la densification ${ }^{22}$. Malgré l'essor de méthodes géomatiques de planification spatiale des

18 Lauren Andres, «Les usages temporaires des friches urbaines, enjeux pour l'aménagement ", op. cit.; Minseo Kim, Christoph Rupprecht et Katsunori Furuya, "Residents' Perception of Informal Green Space. A Case Study of Ichikawa City, Japan ", Land, vol. 7, n 3, 2018, https://www.mdpi. com/2073-445X/7/3/102, consulté le 15 juin 2016; Peleg Kremer, Zoe Hamstead et Timon McPhearson, "A Social-Ecological Assessment of Vacant Lots in New York City ", Landscape and Urban Planning, $\mathrm{n}^{\circ}$ 120, 2013, p. 218-33.

19 Lauren Andres, "Temps de veille de la friche urbaine et diversité des processus d'appropriation : la Belle de Mai (Marseille) et le Flon (Lausanne) ", Géocarrefour, vol. 81, no 2, 2006, p. 159-166; Katherine Foo et al., "The Production of Urban Vacant Land: Relational Placemaking in Boston, MA Neighborhoods ", Cities, n 40, 2014, p. 175-82; Jennifer Foster, " Hiding in Plain View: Vacancy and Prospect in Paris' Petite Ceinture ", Cities, $\mathrm{n}^{\circ} 40$, 2014, p. 124-132; Paula Orduña-Giró et Sébastien Jacquot, "La production participative d'espaces publics temporaires en temps de crise. Le projet "Pla Buits" à Barcelone ", Métropolitiques, 7 novembre 2014, https://www.metropolitiques. eu/La-production-participative-d.html, consulté le 31 décembre 2017.

Raffaele Lafortezza et al., "Visual Preference and Ecological Assessments for Designed Alternative Brownfield Rehabilitations ", Journal of Environmental Management, vol. 89, n 3, p. 257-69.

21 Charles Ambrosino et Lauren Andres, «Friches en ville : du temps de veille aux politiques de l'espace ", Espaces et sociétés, vol. 3, n 134, 2008, p. 37-51; Philippe Bachimon, "Paradoxales friches urbaines ", L'information géographique, vol. 78, n 2, 2014, p. 42-61; Émeline Bailly, « Franges intra-urbaines à l'épreuve des projets de paysage ", Projets de Paysage, 2016, http://www. projetsdepaysage.fr/fr/_franges_intra_urbaines_1_preuve_des_projets_de_ paysage, site consulté le 2 septembre 2018; Luc Lévesque, « Montréal, l'informe urbanité des terrains vagues pour une gestion créatrice du mobilier urbain ", Les Annales de la recherche urbaine, $\mathrm{n}^{\circ}$ 85, 1999, p. 47-57.

Takeshi Kobayashi et Shinji Ikaruga, " Occurrence Factors of Large Vacant Lots in Central Districts and Their Utilization by Local Governments in Japan ", Frontiers of Architectural Research, vol. 5, no 4, 2016, p. 393-402; Lisa Pizzol et al., «Timbre Brownfield Prioritization Tool to Support Effective Brownfield Regeneration ", Journal of Environmental Management, 2016, $\mathrm{n}^{\circ}$ 166, p. 178-92; Béatrice Plottu et Majorie Tendero, "Acceptabilité des choix d'aménagement des friches urbaines polluées ", Revue d'économie régionale et urbaine, $\mathrm{n}^{\circ} 2,2017,335-353$. 
délaissés ${ }^{23}$, l'urbanisation des délaissés se fait très souvent aux dépens de leur intérêt écologique ${ }^{24}$ ou plus généralement leur intérêt naturel, comme le montre une synthèse bibliographique récente $^{25}$. Ceci est à nuancer par le fait que parfois les délaissés évoluent vers des espaces verts aménagés, quiil s’agisse de parcs urbain ${ }^{26}$ ou de jardins collectifs ${ }^{27}$. Sur les plans écologique et social les

23 Alistair Beames et al., «Amenity Proximity Analysis for Sustainable Brownfield Redevelopment Planning ", Landscape and Urban Planning, n' 171, 2017, p. 68-79; Maria Chrysochoou et al., "A GIS and Indexing Scheme to Screen Brownfields for Area-Wide Redevelopment Planning ", Landscape and Urban Planning, $\mathrm{n}^{\circ}$ 105, 2012, p. 187-98; Harriet Herbst et Volkmar Herbst, "The Development of an Evaluation Method Using a Geographic Information System to Determine the Importance of Wasteland Sites as Urban Wildlife Areas ", Landscape and Urban Planning, vol. 77, $\mathrm{n}^{\text {os }}$ 1-2, 2006, p. 178-195; Michael R. Thomas, "A GIS-Based Decision Support System for Brownfield Redevelopment ", Landscape and Urban Planning, vol. 58, n 1, 2002, p. 7-23.

24 Carolyn Harrison et Gail Davies, « Conserving Biodiversity That Matters: Practitioners' Perspectives on Brownfield Development and Urban Nature Conservation in London ", Journal of Environmental Management, vol. 65, $\mathrm{n}^{\circ} 1,2002$, p. $95-108$.

25 Christine Haaland et Cecil Konijnendijk van den Bosch, "Challenges and Strategies for Urban Green-Space Planning in Cities Undergoing Densification: A Review», Urban Forestry and Urban Greening, vol. 14, n 4, 2015, p. 760-771.

26 Ségolène Darly, Pauline Marty et Johan Milian, "La "nature en ville" à l'épreuve de la requalification des banlieues. Le cas de Plaine-Commune ", 20 novembre 2013, p. 1-https://www.metropolitiques.eu/La-nature-en-villea-l-epreuve-de.html, consulté le 12 février 2018; Christopher A. De Sousa, " Turning Brownfields into Green Space in the City of Toronto ", Landscape and Urban Planning, $\mathrm{n}^{\circ}$ 62, 2003, p. 181-198; Kieron J. Doick et al., "Understanding Success in the Context of Brownfield Greening Projects: The Requirement for Outcome Evaluation in Urban Greenspace Success Assessment ", Urban Forestry and Urban Greening, vol. 8, n 3, 2009, p. 16378; Ingo Kowarik, «The "Green Belt Berlin”: Establishing a Greenway Where the Berlin Wall Once Stood by Integrating Ecological, Social and Cultural Approaches ", Landscape and Urban Planning, ${ }^{\circ}$ 184, 2019, p. 12-22; Ingo Kowarik et Andreas Langer, « Natur-Park Südgelände: Linking Conservation and Recreation in an Abandoned Railyard in Berlin ", dans Ingo Kowarik et Stefan Körner (dir.), Wild Urban Woodlands: New Perspectives for Urban Forestry, Berlin, Springer-Verlag, 2005, p. 287-299; Ebba Lisberg Jensen et Pernilla Ouis, "Contested Construction of Nature for City Fringe Outdoor Recreation in Southern Sweden. The Arrie Case ", Urban Forestry and Urban Greening, vol. 7, n 3, 2008, p. 171-82.

27 Luke Drake et Laura J. Lawson, "Validating Verdancy or Vacancy? The Relationship of Community Gardens and Vacant Lands in the U.S. ", Cities, 
délaissés urbains sont pourtant bien différents des espaces verts aménagés et constituent une nouvelle forme de nature en ville ${ }^{28}$. Toutefois, malgré cet intérêt pour les délaissés urbains en urbanisme, les représentations des urbanistes et gestionnaires sur les délaissés ont été peu étudiées ${ }^{29}$.

Suite à l'analyse des usages et des représentations des délaissés urbains par les habitants ${ }^{30}$ l'objectif du travail présenté ici est d'explorer les représentations des gestionnaires de ces espaces, et de les comparer à celles des habitants. Nous faisons l'hypothèse que les représentations des gestionnaires sont influencées par leur potentialité d'action sur les délaissés, en distinguant les personnes publiques non propriétaires de délaissés, des propriétaires de délaissés. Nous questionnons également les représentations comparées des gestionnaires et des habitants du caractère « naturel » (naturalité) des délaissés.

\section{Méthodologie}

Les travaux sur les usages et représentations des délaissés dans les villes moyennes sont peu nombreux. Pourtant ces dernières, particulièrement touchées par l'étalement urbain, constituent un

$\mathrm{n}^{\circ}$ 40, 2014, p. 133-42; Katherine Foo et al., op. cit.

28 Dieter Rink et Harriet Herbst, "From Wasteland to Wilderness. Aspects of a New Form of Urban Nature ", dans Matthias Richter and Ulrike Weiland (dir.), Applied Urban Ecology: A Global Framework, Chichester, West Sussex (UK), Wiley-Blackwell, 2012, p. 82-92; Caragh G. Threlfall et Dave Kendal, "The Distinct Ecological and Social Roles That Wild Spaces Play in Urban Ecosystems ", Urban Forestry and Urban Greening, n 29, 2017, p. 1-9.

Mathias Hofmann et al., "Perceptions of Parks and Urban Derelict Land by Landscape Planners and Residents ", Urban Forestry and Urban Greening, vol. 11, n 3, 2012, p. 303-312.

30 Marion Brun et al., "Les services de support de diversité floristique rendus par les délaissés urbains ", op. cit:; Marion Brun, Francesca Di Pietro et Sébastien Bonthoux, "Residents' Perceptions and Valuations of Urban Wastelands Are Influenced by Vegetation Structure ", Urban Forestry and Urban Greening, n² 29, 2018, p. 393-403; Marion Brun et al., "Usages et représentations des délaissés urbains, supports de services écosystémiques culturels en ville ", Environnement Urbain / Urban Environment, n' ${ }^{\circ}$ 11, 2017, http://journals.openedition.org/eue/1906, consulté le 12 juin 2012. 
paysage complexe, à l'interface entre métropole et campagne. C'est le cas notamment des villes ligériennes ${ }^{31}$, parmi lesquelles nos sites d'étude : les agglomérations de Tours et Blois.

\section{Échantillonnage des enquêtés}

Pour ces enquêtes, nous avons suivi une méthodologie différente pour chaque type d'acteurs : (1) gestionnaires et (2) habitants riverains des délaissés. Alors que nous nous sommes fondés sur la liste de propriétaires des délaissés pour l'identification des gestionnaires, nous avons sélectionné des habitants riverains des délaissés en effectuant des enquêtes à proximité immédiate d'un sous-échantillon de délaissés, ce qui permet aux habitants d'exprimer non seulement leurs représentations, relevant de la cognition, mais également leurs perceptions, émergées en présence de l'objet ${ }^{32}$.

Les différents gestionnaires interrogés sont des personnes publiques non propriétaires de délaissés, ayant potentiellement une action de gestion sur ces espaces (élus, agences d'urbanisme des collectivités, $n=6$ ), que nous allons nommer " collectivités locales " par la suite, et des propriétaires de délaissés $(n=23$, pour 60 délaissés en tout). Ces derniers sont des propriétaires publics (état, mairies, communautés d'agglomération, etc., $\mathrm{n}=12$ ), des propriétaires aménageurs (sociétés d'économie mixte, offices publics de l'habitat, $\mathrm{n}=3$ ) ou des propriétaires privés, entreprises $(n=4)$ ou particuliers $(n=4)$. Au total, 29 gestionnaires ont été interrogés.

Les enquêtes auprès des habitants ont ciblé 18 délaissés urbains, 9 dans chaque site, sélectionnés en fonction de leur place dans le gradient urbain (trois classes de distances au centre-ville : centre-ville, suburbain, périurbain) et présentant les trois stades successionnels de la végétation observés (stade primaire : pelouse;

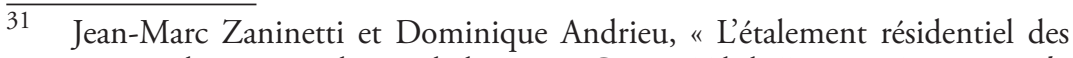
métropoles intermédiaires de la région Centre Val de Loire ", Mappemonde, $\mathrm{n}^{\circ}$ 124, 2018, p. 1-21.

32 Émeline Bailly, op. cit:: Lucile Grésillon, Sentir Paris. Bien-être et matérialité des lieux, Versailles, Quae, coll. "Indisciplines ", 2010. 
stade intermédiaire : herbes hautes; stade avancé : milieu buissonnant; figure 1). Aucun délaissé n'est clôturé. Quatre personnes par délaissé, soit 72 habitants au total ont été interrogées par entretien.

Figure 1. Photographies de l'aspect de la végétation des délaissés catégorisée selon trois stades d'avancement et utilisée comme critère de sélection pour les enquêtes

a.



Stale primaire

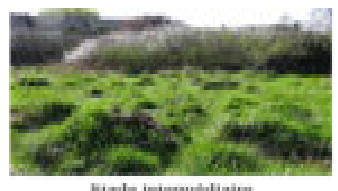

siade iniermethaire

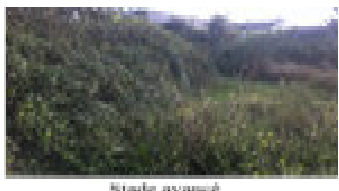

Stade avanes

b.



L'ensemble de la démarche d'échantillonnage est présenté dans le tableau 1.

Tableau 1. Synthèse des enquêtes effectuées auprès des gestionnaires et habitants

\begin{tabular}{ll|l|l}
\hline \multicolumn{3}{c|}{ Gestionnaires $(\mathbf{n}=\mathbf{2 9})$} & $\begin{array}{l}\text { Habitants riverains } \\
\text { des délaissés }(\mathbf{n}=\mathbf{7 2})\end{array}$ \\
\hline $\begin{array}{l}\text { Nombre } \\
\text { d'acteurs } \\
\text { interrogés }\end{array}$ & $\begin{array}{l}\text { propriétaires }(\mathrm{n}=23): \\
\text { publics }(\mathrm{n}=12), \\
\text { aménageurs }(\mathrm{n}=3), \text { privés } \\
(\mathrm{n}=8)\end{array}$ & $\begin{array}{l}\text { non propriétaires : } \\
\text { collectivités locales } \\
(\mathrm{n}=6)\end{array}$ & $\begin{array}{l}\text { 4 habitants/délaissé sur } \\
18 \text { délaissés }\end{array}$ \\
\hline $\begin{array}{l}\text { Délaissés } \\
\text { concernés }\end{array}$ & $\begin{array}{l}\text { de manière générale et sur } \\
\text { le(s) délaissé(s) dont la } \\
\text { personne est propriétaire }\end{array}$ & de manière générale & $\begin{array}{l}\text { sur un délaissé en } \\
\text { particulier }\end{array}$ \\
\hline
\end{tabular}


Recueil et analyse des données

Concernant les gestionnaires, la méthode des entretiens semidirectifs convient pour effectuer des études approfondies ${ }^{33}$. Concernant les habitants, l'outil d'enquête défini comme le plus pertinent dans ce cadre est l'entretien directif, qui permet de recueillir un maximum d'informations facilement comparables en peu de temps (10-15 minutes $\left.{ }^{34}\right)$. Pour les deux populations les enquêtes ont été centrées sur (i) les activités observées, réalisées ou imaginées sur les délaissés (usages, pratiques, projets d'aménagement futur de l'espace), (ii) les représentations de ces espaces (de manière générale et concernant les représentations de la naturalité) et (iii) l'attribution de valeurs à des critères prédéfinis présentés dans un tableau pour caractériser rapidement l'espace (tableau 2).

Tableau 2. Liste de critères proposée aux enquêtés : il était demandé d'attribuer une note entre deux formulations antinomiques de chaque critère. Prenons l'exemple du critère d'utilité : de part et d'autre du tableau sont écrits les critères " inutiles" (proche de la note -2 ) et « utiles " (proche de la note +2$)$. Si la personne trouve l'espace complètement inutile : elle coche la case -2 , si elle trouve l'espace complètement utile : elle coche la case +2 , si elle a un avis neutre sur la question : elle coche 0 .

\begin{tabular}{l|lllll|l}
\hline critère & -2 & -1 & 0 & 1 & 2 & critère antinomique \\
\hline artificiel & & & & & & naturel \\
dompté & & & & & & sauvage \\
inintéressant & & & & & & intéressant \\
désagréable & & & & & agréable \\
repoussant & & & & & attrayant \\
inutile & & & & & utile \\
négligé & & & & & entretenu \\
\hline
\end{tabular}

33 Rodolphe Ghiglione et Benjamin Matalon, Les enquêtes sociologiques. Théories et pratique, Paris, Armand Colin, coll. « U : sociologie », 1998. Ibid. 
Les réponses des enquêtés ont fait l'objet d'une analyse thématique visant à l'interprétation du contenu ${ }^{35}$ et certaines ont été soumises à des tests d'indépendance (tests $\mathrm{du} \mathrm{Chi}^{2}$ ), afin de déterminer les principales relations qui s'établissent entre les réponses des enquêtés selon les thèmes abordés.

\section{Résultats}

Usages des délaissés

\section{Les projets sur les délaissés par les gestionnaires}

Les gestionnaires interrogés prennent en compte ces espaces de manière ponctuelle dans la planification du territoire. Ils évoquent de manière spontanée les délaissés comme espaces de projet (pour $50 \%$ d'entre eux), parfois, dès le début de l'entretien, lorsqu'ils définissent ce qu'est pour eux un délaissé. Une majorité des gestionnaires interrogés déplore l'impossibilité récurrente de mettre en œuvre des projets sur ces terrains. Dans cette optique nous avons abordé, avec les gestionnaires ayant des réflexions ou actions en aménagement (soit 17 interrogés, dans les catégories propriétaires publics, propriétaires aménageurs et collectivités locales), la question du projet, depuis l'inclusion du délaissé dans une démarche de planification ou d'aménagement jusquà la construction du terrain.

En ce qui concerne la démarche de planification, l'intégralité des propriétaires publics et des propriétaires aménageurs considère que le délaissé n'est pas inclus dans une réflexion de projet. En revanche les représentants des collectivités locales ont des propos plus nuancés : $75 \%$ d'entre eux estiment avoir mis en place des réflexions ou des actions (de gestion ou d'aménagement) propres à leur prise en compte $(25 \%$ considèrent ne pas en avoir mis en place).

$\overline{35}$ Bernard Fallery et Florence Rodhain, «Quatre approches pour l'analyse de données textuelles ", $\mathrm{XVI}^{\mathrm{e}}$ Conférence de l'Association Internationale de Management Stratégique, Montréal, Canada, 2007, p. 1-16, https://hal. archives-ouvertes.fr/hal-00821448, consulté le 16 août 2018. 
Les projets envisagés par les propriétaires sur les délaissés qu'ils possèdent sont majoritairement la construction (46\%, figure 2 ), ou la cession et commercialisation du terrain ( $24 \%$, dans certains cas la vente était déjà réalisée au moment de l'enquête). Ces projets sont essentiellement présents dans le discours des propriétaires aménageurs, dont le travail consiste à aménager des terrains pour les rendre constructibles (équipements, réseaux...) et à les vendre à des promoteurs. L'ensemble des propriétaires évoquent également le développement ou le maintien d'une activité, qu'elle soit industrielle ou de service. Une minorité d'entre eux a évoqué " un parc ", " la mise en place d'une culture » ou " l'installation d'équipements sportifs », ces choix de projet étant pour la plupart liés à la localisation du délaissé en zone inondable. Les propriétaires privés particuliers évoquent notamment la difficulté de revendre ou requalifier des terrains non constructibles, ce qui repousse tous les potentiels investisseurs. Une autre minorité de gestionnaires n'a pas encore déterminé un usage définitif du terrain (" divers »: $7 \%$ ). Ils le gardent donc en état de délaissé (figure 2).

Figure 2. Projets prévus sur les délaissés

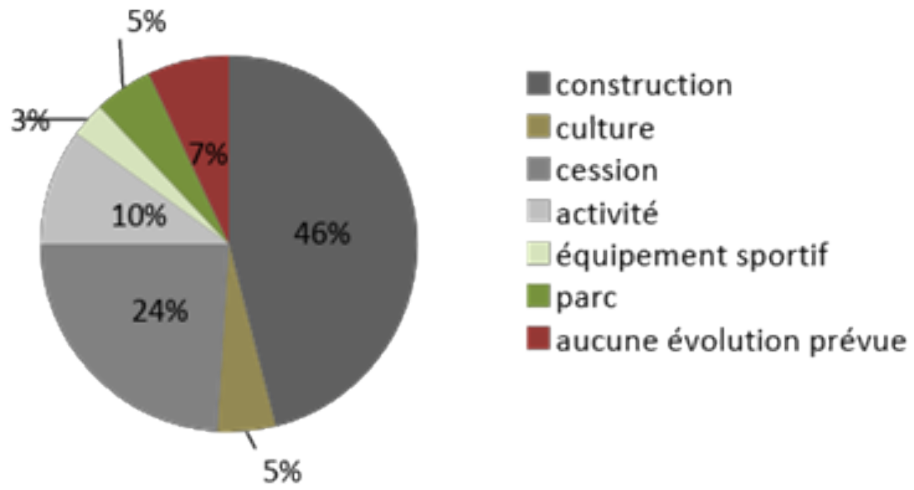

Pour l'ensemble de ces gestionnaires il existe donc deux types principaux de délaissés : ceux qui sont en attente de projet, avec un temps de mise en ouvre incompressible du fait de contraintes administratives et réglementaires, et les délaissés sur lesquels il 
n'est pas possible de faire un projet, pour diverses raisons, notamment le caractère non constructible de l'espace (zone inondable, servitude particulière).

Les usages des délaissés : pratiques des habitants et attitudes des gestionnaires

Comme cela a été montré dans l'étude centrée sur les habitants riverains des délaissés ${ }^{36}$, une majorité d'habitants interrogés ne fréquente pas le délaissé, estimant n'avoir rien à y faire (64\%). En revanche, pour les $36 \%$ des habitants qui fréquentent ces espaces plusieurs fois par semaine, différents usages sont énoncés. Le délaissé est utilisé comme lieu de promenade (13\% des réponses), notamment pour promener son chien. Ces espaces sont aussi considérés comme des lieux de détente et d'activités de plein-air (8\% des réponses; divers sports ont été évoqués : pétanque, cerf-volant, etc.) et de jeu pour les enfants $(15 \%$ des réponses). La majorité des personnes fréquentant l'espace indique également de multiples usages potentiels, autres que ceux pour lesquels elles utilisent habituellement l'espace, regroupant des activités de promenade, des jeux pour les enfants, etc. (26\% des réponses).

Concernant les gestionnaires, nous avons constaté que $81 \%$ des gestionnaires propriétaires ont autorisé ou constaté des usages temporaires sur leur(s) terrain(s). Comme l'illustre la figure 3, parmi ces derniers figurent des usages illicites, tels que le squat par des gens du voyage, qui est le principal usage évoqué (32\%), ou le dépôt de déchets (9\%). Toutefois ces usages sont temporaires et n'impactent que très rarement le projet envisagé sur le délaissé : les acteurs rencontrés ont mentionné que des discussions avec les "squatteurs " suffisaient pour qu’ils quittent le délaissé, sans que cela remette en question, s'il y en a, un projet. Le déversement de déchets sur site représente davantage un agacement et un coût pour le propriétaire qu'une réelle remise en question du projet.

36 Marion Brun et al., «Usages et représentations des délaissés urbains, supports de services écosystémiques culturels en ville ", op. cit. 
Figure 3. Principaux usages temporaires énoncés par les gestionnaires ayant autorisé ou constaté des usages temporaires sur leurs terrains (autre : parking, lieu de stockage)

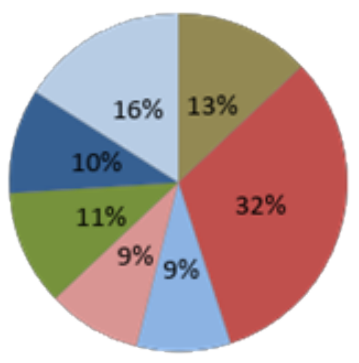

Convention avec un agriculteur squat / gens du voyage Artistes / Associations Dépôt de déchets Jardin / Prairie fleurie Détente / promenade / jeu $\square$ Autre

D’autres usages sont autorisés par les propriétaires de délaissés; certains propriétaires publics passent une convention avec des agriculteurs pour l'entretien du terrain (13\%). L'agriculteur dispose alors, souvent gratuitement, de l'espace. Le semis de prairies fleuries, considéré par les propriétaires interrogés comme un usage, ou la mise en place de jardins familiaux sur les délaissés sont également énoncés (11\%). Selon les propriétaires, ces usages sont perçus positivement par les habitants, ce qui permet une réflexion sereine quant au devenir du terrain, car cela «n'accentue pas son image négative » (entretien 14). Il arrive également que des conventions soient passées avec des associations ou artistes dans un souci de promotion de la culture au niveau local : "L'autorisation d'usages temporaires, en particulier des associations ou artistes, permet de faire connaître le quartier, de faire de la publicité sur l'usage final du délaissé [...] ça nous avait permis de dire il va se passer des choses ici ou il s'en passe toujours " (entretien 11).

Les usages temporaires, autorisés ou non par le propriétaire, n'affectent donc pas l'usage définitif du terrain, quand celui-ci est déjà prévu en amont. En effet, lorsqu'un usage temporaire a été cité par les personnes interrogées, il n’a jamais été question de le rendre définitif. Pour cela, la communication auprès des habitants est primordiale : "On a toujours communiqué sur des 
opérations où les gens savaient bien que c'était du provisoire et que derrière on allait transformer ces délaissés en opérations d'habitat " (entretien 14).

Nous avons montré l'existence d'usages multiples sur les délaissés. Ces espaces sont le support de pratiques urbaines parfois informelles. Bien qu'une partie de ces usages ne soient pas autorisés, les gestionnaires n'ont pas manifesté d'inquiétude quant à la réalisation de projets sur ces espaces.

Représentations des délaissés

Par les gestionnaires

L'analyse du discours des gestionnaires permet de faire ressortir certaines notions impliquées dans la définition des délaissés. Ces espaces sont principalement définis comme abandonnés (ce qui est mentionné de manière explicite par 16 répondants sur 29 et de manière implicite par 6 répondants sur 29). D’autres répondants considèrent en revanche que l'absence d'usage n' est pas synonyme d'un abandon, mais que cela laisse la place à d'autres représentations, comme la nature. Le caractère végétal est mentionné spontanément pour 18 interrogés et est intégré à la définition des délaissés. La temporalité est également une notion très présente dans la définition des délaissés par les gestionnaires. 18 répondants sur 29 mentionnent spontanément leur caractère temporaire au sein du projet urbain. Neuf gestionnaires évoquent par ailleurs le manque de projet sur ces espaces. Ainsi, l'état transitoire des délaissés au sein des projets urbains est une composante majeure de leur définition.

Aux yeux des gestionnaires, les délaissés urbains sont des espaces présentant de la végétation ou des éléments bâtis qui avaient une fonction dans le territoire, aujourd'hui perdue. Ils sont considérés comme abandonnés, ce sont des " espacesproblème » qui néanmoins peuvent être porteurs d'opportunités de projet, si les acteurs s'en saisissent. Cette définition consensuelle peut être nuancée selon le type de gestionnaire. Pour les représentants des collectivités locales, ces espaces sont abandonnés, à fort potentiel pour des projets. Pour les propriétaires publics et 
les propriétaires aménageurs, les délaissés présentent un fort potentiel mais sont une contrainte à gérer. Pour les propriétaires privés particuliers, c'est un espace-problème du fait de règles d'urbanisme contraignantes. En revanche les propriétaires privés entreprises ne voient pas le délaissé comme une opportunité, ils n’ont en général pas de perspectives pour ces espaces. À l'exception de certaines collectivités locales, les gestionnaires interrogés considèrent que les délaissés véhiculent une image négative (27 sur 29). Diverses raisons de cette représentation négative ont été évoquées. En premier lieu, les délaissés sont inesthétiques pour $80 \%$ des propriétaires. Par ailleurs, la végétation envahissante déplait aux riverains, ces espaces sont vus comme envahis et suggèrent un manque d'entretien. Les délaissés véhiculent également en majorité une image négative car ils représentent une " erreur d'aménagement » et symbolisent un problème dans la planification de l'espace. Ceci ressort de manière forte pour les propriétaires particuliers, qui évoquent tous une "erreur d'aménagement". Diverses autres causes des représentations négatives ont été identifiées, comme la peur du danger, du squat, des plantes et animaux indésirables ou, enfin, les coûts d'entretien.

Les représentations de la naturalité des délaissés urbains dépendent du type de gestionnaire. Les propriétaires privés (particuliers et entreprises) considèrent majoritairement le délaissé comme un espace naturel; ils estiment que le délaissé est un espace où la nature reprend ses droits, la végétation s'y développe malgré un milieu (urbain) qui n'est pas a priori favorable. Ces acteurs-là mentionnent aussi la végétation abondante ou le passé agricole du délaissé. En revanche, les propriétaires aménageurs pensent majoritairement que le délaissé est un espace artificiel du fait des anciennes activités anthropiques. Ils se réferent à l'utilisation précédant le délaissé pour déterminer le degré de naturalité. Parmi les propriétaires publics, certains considèrent que les délaissés sont naturels, de la même manière que les propriétaires privés, d'autres ont une opinion contradictoire sur la naturalité du délaissé, mettant en lumière tantôt le caractère naturel (la végétation, se développant sur un terrain abandonné depuis 
plusieurs années, est associée à l'aspect naturel des délaissés), tantôt le caractère artificiel (les interventions anthropiques, notamment l'occupation de terrains par des activités industrielles, étant vues comme à l'origine du caractère artificiel des délaissés : l'activité humaine, passée ou présente, diminue alors le degré de naturalité du délaissé). Enfin, les représentants des collectivités locales n'évoquent pas spontanément le caractère naturel ou artificiel du délaissé. Ils en font mention seulement quand on leur demande clairement de choisir l'un ou l'autre de ces qualificatifs dans le tableau des critères : pour eux, la naturalité n'est pas un élément à prendre en compte pour la définition de ces espaces.

\section{Par les habitants}

Les représentations des délaissés de la part des habitants sont présentées dans la figure 4. Une majorité d'habitants voit le délaissé comme un "lieu abandonné " (51\% des réponses). L'idée d'abandon émerge régulièrement des enquêtes, en relation aux terrains non entretenus par leur propriétaire. Dans une moindre proportion, des habitants mentionnent un espace vert (14\%), ce qui fait appel à la naturalité du lieu (détaillée par la suite). On trouve également les termes « décharge », liée à l'usage, et "terrain vague " $(12 \%$ et $8 \%$ respectivement). Les représentations des habitants renvoient également au manque de projet urbain : pour $11 \%$ d'entre eux les délaissés sont voués à disparaître, ils sont " en attente ", caractérisés par leur vocation future. Nous pouvons donc souligner que la vision des habitants semble proche de celle des gestionnaires en ce qui concerne l'abandon et le manque d'utilisation pour le projet. 
Figure 4. Représentations du délaissé par les habitants

Qu'est ce que cet espace représente pour vous?



Terrain en attente

Décharge

Espace vert

Lieu abandonné

Terrain vague

Autre

Source : Marion Brun et al., " Usages er représentations des délaissés urbains, supports de services écosystémiques culturels en ville ", op. cit.)

L'évaluation générale, positive, négative ou neutre des délaissés par les habitants est en relation avec leurs représentations : l'évaluation positive du délaissé est liée notamment à sa représentation comme un espace vert, l'évaluation négative étant liée notamment à sa représentation comme décharge (figure 5).

Le statut transitoire des délaissés, exprimé à travers les représentations, peut provoquer, parmi les habitants riverains, une incompréhension de leur place au sein de la ville (figure 5) et la végétation peut en être le reflet. À l'inverse, aux yeux d'autres habitants, cette végétation répond à un besoin de nature en ville : comme l'indique la figure 4, $14 \%$ d'entre eux considèrent le délaissé comme un espace vert et, tout en considérant cet espace comme terrain vague ou abandonné, soulignent son caractère naturel. Ces derniers apprécient alors l'absence d'entretien et le caractère spontané, libre de la végétation. Ces espaces sont qualifiés de " coin de verdure " ou de " rideau végétal " (habitants à proximité des délaissés $n^{\circ} 10$ et $n^{\circ} 50$ ). Pour certains, un espace naturel est un espace géré, le délaissé est alors apprécié et le développement de la végétation n'est pas perçu négativement : parmi les habitants considérant le délaissé comme un espace vert (donc naturel), 91,7 \% l'estiment de manière positive (figure 5). 
De la même manière, les habitants considérant le délaissé comme un terrain vague l'apprécient majoritairement.

Figure 5. Relation entre l'évaluation générale du délaissé et ses représentations. Les diagrammes indiquent les réponses des habitants à la question : "Qu' est-ce que cet espace représente pour vous? ", selon l'évaluation - positive, négative ou neutre - des habitants sur le délaissé en question.



Source : Marion Brun et al., " Usages er représentations des délaissés urbains, supports de services écosystémiques culturels en ville ", op. cit.)

Les représentations des habitants se rapprochent de celles des gestionnaires concernant l'abandon et le manque de projet sur les délaissés, en revanche un caractère positif, que l'on ne retrouve pas chez les gestionnaires, est associé aux délaissés considérés comme des espaces verts. Nous soulignons également qu'il y a un lien significatif entre l'image positive et l'usage de cet espace (test $\mathrm{du} \mathrm{Chi}^{2}$ : P-Value $<0,001$ ) : les habitants se représentent les délaissés de manière positive lorsqu'ils y effectuent des usages divers $^{37}$.

Les délaissés sont considérés comme étant des espaces naturels pour environ la moitié des habitants (35 sur 72 : figure 6). Ce caractère naturel est associé à des représentations contrastées, liées à l'image spontanée et non entretenue de la végétation. Par ailleurs, l'aspect de la végétation (représenté par le stade de végétation)

37 Marion Brun et al., « Usages et représentations des délaissés urbains, supports de services écosystémiques culturels en ville ", op. cit. 
influence la représentation de la naturalité et l'appréciation qui en découle. Le stade intermédiaire de végétation est considéré comme le plus naturel et est le plus apprécié $\left(\mathrm{Chi}^{2}, \mathrm{P}-\mathrm{Value}<0,01\right)^{38}$. À l'inverse, le stade " primaire " de végétation ne renvoie pas une image d'espace de nature. Une végétation trop basse n'est donc pas considérée comme naturelle et une végétation trop avancée est un frein à l'appréciation du délaissé (figure 6).

Figure 6. Relation entre la représentation de la naturalité du délaissé et le stade de la végétation. Les diagrammes indiquent les réponses des habitants à la question "Considérez-vous cet espace comme naturel? ", selon le stade de végétation du délaissé en question.



Les valeurs des délaissés : comparaison des représentations des gestionnaires et des habitants

Les valeurs attribuées aux critères de qualification des délaissés par les gestionnaires propriétaires de délaissés et par les habitants nous permettent de comparer les représentations de ces deux types d'acteurs. Les gestionnaires considèrent essentiellement les délaissés selon leur utilité (figure 7) : ces derniers ont donné en majorité des notes positives au critère " utile " pour désigner les délaissés et seuls 6 sur 23 considèrent les délaissés comme étant " inutiles»(note de -2 au critère d'utilité). Les délaissés sont considérés comme utiles pour mettre en place un projet d'aménagement. D'ailleurs les gestionnaires qualifient ces espaces d'intéressants, mais pas forcément d'attrayants. Leur caractère non entretenu ressort également.

38 Marion Brun et al., ibid. 
Figure 7. Valeurs attribuées par les gestionnaires propriétaires de délaissés $(\mathrm{n}=23$ sur 29$)$ aux critères proposés



Concernant l'aspect des délaissés, ils sont considérés comme naturels pour une majorité de gestionnaires (13 sur 23), mais ne sont pas pour autant agréables et beaux. Ces espaces "font désordre ", ils ne sont ni véritablement entretenus ni agréables (il est souvent mentionné que c'est à cause de la végétation) mais ils sont utiles en aménagement du territoire.

Les réponses des habitants à ce même tableau de critères à propos des délaissés qu'ils avaient en face d'eux font ressortir de grandes différences par rapport aux réponses des gestionnaires propriétaires (figure 8). Comme l'indiquent les entretiens, les délaissés sont considérés par les habitants comme des espaces naturels, ils sont aussi considérés comme sauvages et intéressants. Le lien entre les caractères naturel et utile des délaissés est attesté : $75 \%$ des habitants considérant le délaissé comme naturel le trouvent également utile $\left(\mathrm{Chi}^{2}, \mathrm{P}\right.$-Value $\left.<0,05\right)$.

On retrouve par ailleurs une majorité d'avis neutres quant aux caractères " agréable » et " attrayant ». Nous pouvons sur ce point noter une majorité d'avis neutres lorsque l'adjectif proposé 
renvoie plus à la relation de la personne avec l'objet qu'à l'objet lui-même (le délaissé est agréable, attrayant pour la personne). À l'inverse, lorsque l'adjectif proposé renvoie à l'objet délaissé en soi, les avis sont plus tranchés (naturel, sauvage, entretenu). Les réponses des habitants sont donc relativement floues lorsqu'il est question d'exprimer leur propre avis sur les délaissés. Ceci est à mettre en parallèle avec la difficulté d'identifier, voire de nommer les délaissés, ces derniers n'ayant a priori pas de fonction propre. Les adjectifs " intéressant " et " utile " sont à ce propos difficiles à interpréter, pouvant renvoyer à la personne, mais également à un groupe (les habitants du quartier, la collectivité).

Figure 8. Attribution de notes par les habitants $(\mathrm{n}=72)$ aux critères proposés

$m-2=-1=0=1=2$

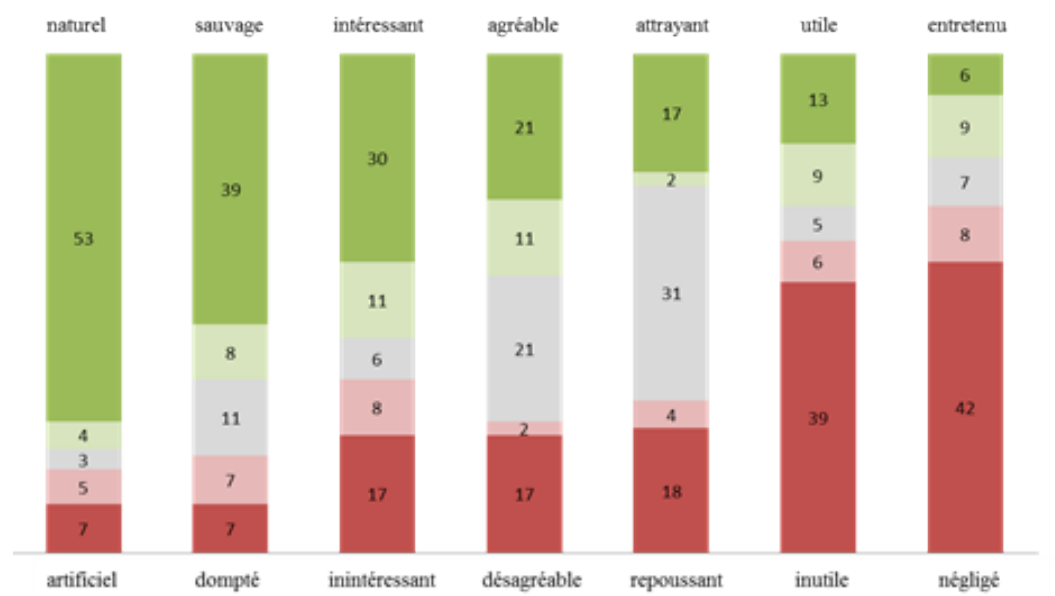

La comparaison des critères énoncés par les deux types d'acteurs nous permet de voir que ces espaces sont utiles aux yeux des gestionnaires, mais pas aux yeux des habitants riverains. Même si tous s'accordent sur le fait que ces espaces sont naturels, non entretenus et intéressants, la représentation des délaissés paraît bien plus positive pour les habitants que pour les propriétaires : de manière générale, les délaissés sont agréables, même s'ils sont peu entretenus et pas vraiment beaux. 


\section{Interprétation et conclusion}

Convergences et divergences dans les représentations des délaissés urbains et de leur naturalité par les gestionnaires et les habitants

À travers les enquêtes réalisées, nous pouvons noter que la représentation des délaissés par les habitants est relativement proche des caractéristiques énoncées par les gestionnaires en ce qui concerne la définition des délaissés. Malgré des points de vue contrastés entre gestionnaires et habitants, les délaissés sont majoritairement représentés comme des espaces non entretenus et végétalisés. Les caractères temporaire et abandonné font également consensus dans la description des délaissés, aussi bien pour les gestionnaires que pour les habitants.

Toutefois, si chez les propriétaires particuliers et certains habitants, l'incertitude qui accompagne la vacance de ces espaces peut provoquer une vision négative des délaissés, qui renvoient une image de déshérence ${ }^{39}$, pour d'autres habitants, ce devenir incertain est, à l'inverse, un point positif, il renvoie à une idée de liberté qui confirme la demande sociale d'espaces de respiration dans la ville de la part de certains habitants ${ }^{40}$.

Les représentations de la naturalité des délaissés par les gestionnaires laissent entrevoir un rapport étroit à leur temporalité. Les gestionnaires propriétaires ne disent pas être propriétaires de délaissés urbains : ces espaces sont, selon eux, pensés dans un projet, ils ne sont donc pas délaissés, ni « naturels ». Tout se passe comme si la nature était perçue en relation à la temporalité : en se référant à un contexte passé, l'acteur interrogé considère le délaissé comme naturel. En se référant à un contexte présent, l'acteur le considère comme à la fois naturel et artificiel. En se référant à un contexte futur, l'acteur considère que le délaissé est artificiel (figure 9).

$39 \quad$ Lauren Andres, "Temps de veille de la friche urbaine et diversité des processus d'appropriation : la Belle de Mai (Marseille) et le Flon (Lausanne) ", op. cit.

40 Jennifer Foster, op. cit. 
Figure 9. La représentation de la naturalité selon le type de gestionnaire : un rapport au temps

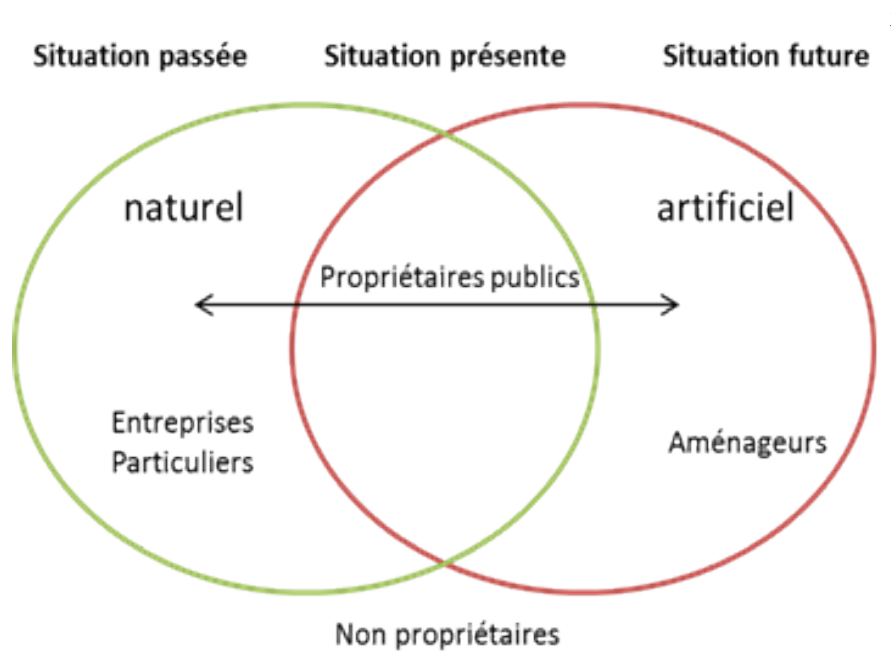

En confrontant notre étude aux travaux qui comparent les représentations des délaissés urbains par les gestionnaires du paysage (landscape planners) et les habitants ${ }^{41}$, nous observons que la préférence des habitants pour une nature domestiquée de type espace vert aménagé est seulement en partie confirmée. La végétation des délaissés est en effet plus appréciée par les habitants lorsqu'elle présente un stade intermédiaire de développement ${ }^{42}$. En revanche, nous ne trouvons pas la préférence des gestionnaires pour des zones naturelles avec une faible accessibilité et une richesse spécifique élevée, indiquée dans l'étude de Mathias Hofmann et ses collaborateurs ${ }^{43}$, probablement parce nous avons interrogés des gestionnaires urbanistes plus que paysagers.

\footnotetext{
41 Mathias Hofmann et al., op. cit.

42 Marion Brun, Francesca Di Pietro et Sébastien Bonthoux, op. cit.

43 Mathias Hofmann, Janneke R. Westermann, Ingo Kowarik, Elke Van der Meer, «Perceptions of parks and urban derelict land by landscape planners and residents", Urban Forestry and Urban Greening, vol. 11, n 3, 2012, p. 303-312.
} 


\section{Donner une visibilité à des espaces en attente}

Les délaissés urbains sont les marges de manœuvre pour une ville compacte vivable et représentent un fort potentiel de construction de la ville sur elle-même dans les dynamiques de densification et renouvellement urbain $^{44}$. Ces espaces en transition, qui ont vocation à disparaître, ne sont que temporaires dans l'espace urbain, ce qui rend difficile leur intégration dans le projet d'aménagement. C'est la raison pour laquelle le délaissé peut être un symbole d'espace gâché : c'est une vision que partagent les propriétaires publics et les propriétaires aménageurs: selon eux, ces espaces ont un fort potentiel, mais présentent des contraintes qui les dévalorisent.

Bien que certains gestionnaires anticipent cette situation et proposent une utilisation provisoire de l'espace, ceci reste rare. Lorsque des projets sont initiés sur un délaissé on peut relever le manque d'information et de communication entre gestionnaires et habitants, et une mise à l'écart de ces derniers. Il nous semble nécessaire que les habitants comprennent la raison du délaissement de ces espaces : une mise en visibilité des perspectives et des projets d'aménagement par les gestionnaires des délaissés aiderait sans doute à mieux apprécier ces espaces durant leur temps de veille, comme le suggèrent d'autres auteurs à propos de l'amélioration de l'appréciation des délaissés par la communication sur leur végétation ${ }^{45}$.

\section{Mettre en forme une nature temporaire}

Les délaissés urbains végétalisés sont emblématiques du « statut hésitant d'une nature ordinaire qui commence à être connue et reconnue, mais qui voisine encore avec le déchet, au sens matériel et symbolique ${ }^{46}$ ». La plupart des habitants apprécient les délaissés

\footnotetext{
44 Cyria Emelianoff, « Urbanisme durable? », Écologie et politique, vol. 29, n 2 , 2004, p. 13-19.

45 Gilles Sénécal et Diane Saint-Laurent, «Espaces libres et enjeux écologiques : deux récits du développement urbain à Montréal ", Recherches sociographiques, vol. 40, n 1, 1999, p. 33-54.

46 Bernadette Lizet, "Du terrain vague à la friche paysagée ", op. cit.
} 
urbains en tant qu'espaces de nature, mais une nature domestiquée; les gestionnaires peuvent accompagner cette préférence, et en même temps contribuer à l'évolution des représentations de la nature spontanée. Pour que le végétal ne soit pas vecteur d'inquiétude, il faudrait une nature " civilisée ${ }^{47}$ ». Konstantinos Tzoulas et Philip James ont évoqué les well kept landscapes ${ }^{48}$, des paysages gérés et structurés par l'homme pour exprimer la préférence des habitants en matière de naturalité de proximité. Ceci est d'autant plus important à prendre en compte en France, où la culture des espaces traditionnels, très domestiqués, des « jardins à la française » reste ancrée dans les représentations collectives des espaces de nature (à la différence des pays anglosaxons, majoritairement représentés dans la littérature internationale). Une étude sensible, appliquée à l'urbanisme, permettrait de créer des espaces adaptés aux besoins de l'homme, qui répondent à une esthétique socialement construite de la nature ${ }^{49}$.

L'appréciation de cette nature urbaine libre nécessiterait un travail de mise en forme, de domestication, qui montre une maîtrise et une gestion humaine visible, reflétant le besoin de domestiquer la nature ${ }^{50}$. Hofmann et ses collaborateurs ont sur ce point montré l'influence de la trace d'une gestion humaine sur la manière dont les habitants se représentent les délaissés ${ }^{51}$. Quelques aménagements légers avec des bancs ou de petites infrastructures peuvent suffire à améliorer l'acceptabilité des délaissés par la population ${ }^{52}$. Des projets initiateurs ont déjà été

47 Christian Calenge, "L'idéologie verte et la rhétorique paysagère ", Communications, $\mathrm{n}^{\circ} 74,2003$, p. 33-47.

48 Konstantinos Tzoulas et Philip James, "Peoples' Use of, and Concerns about, Green Space Networks: A Case Study of Birchwood, Warrington New Town, UK », Urban Forestry and Urban Greening, vol. 9, n 2, 2010, p. 121-128.

49 Gabriel Moser et Karine Weiss, Espaces de vie. Aspects de la relation hommeenvironnement, Paris, Armand Colin, 2003.

50 Bernadette Lizet, "Naturalistes, herbes folles et terrains vagues ", Ethnologie française, vol. 19, n $\mathrm{n}^{\circ}$, 1989, p. 253-272.

51 Mathias Hofmann et al., op. cit.

52 Emily Lorance Rall et Dagmar Haase, «Creative Intervention in a Dynamic City: A Sustainability Assessment of an Interim Use Strategy for Brownfields in Leipzig, Germany ", Landscape and Urban Planning, vol. 100, $\mathrm{n}^{\circ}$ 3, 2011, p. 189-201. 
menés pour faire des délaissés de véritables espaces verts. C'est le cas, par exemple, à Berlin ${ }^{53}$ ou encore à Nantes ${ }^{54}$. À côté de la végétation spontanée ont été mis en place des caillebotis, des bancs, des panneaux botaniques et quelques espaces de jeu, qui révèlent alors l'intérêt de ces espaces pour les services culturels, notamment de loisir, de bien-être et de détente. Certains gestionnaires interrogés ont d'ailleurs déjà mis en œuvre des projets à caractère environnemental sur des délaissés. Plusieurs expérimentations montrent l'intérêt d'une gestion adaptative et temporaire des délaissés par les pouvoirs publics : la modulation des impôts fonciers en échange d'un entretien léger de la végétation des délaissés à Leipzig, Allemagne ${ }^{55}$; le pré-verdissement à grande échelle des friches urbaines qui vont être urbanisées ${ }^{56}$. Les délaissés urbains sont l'opportunité d'offrir aux habitants des espaces de nature alternatifs à l'approche traditionnelle du parc; ils invitent à changer le regard face à la nature spontanée.

53 Leonie K. Fischer, Moritz Von der Lippe et Ingo Kowarik, « Urban Land Use Types Contribute to Grassland Conservation: The Example of Berlin ", Urban Forestry and Urban Greening, vol. 12, n 3, 2013, p. 263-272. Sébastien Bonthoux et al., op. cit.

55 Emily Lorance Rall et Dagmar Haase, op. cit.

56 Guillaume Lemoine, "Usages temporaires des friches urbaines de l'Établissement Public Foncier Nord-Pas-de-Calais : une contribution aux villes durables? ", Techniques, sciences, méthodes, n 3, 2017, p. 1-8. 


\section{Bibliographie}

Ambrosino, Charles et Lauren Andres, "Friches en ville : du temps de veille aux politiques de l'espace ", Espaces et sociétés, vol. 3, n 134, 2008, p. 37-51.

Andres, Lauren, «Les usages temporaires des friches urbaines, enjeux pour l'aménagement ", 2011, https://www.metropolitiques.eu/Les-usagestemporaires-des-friches.html, site consulté le 2 septembre 2018.

Andres Lauren, "Temps de veille de la friche urbaine et diversité des processus d'appropriation : la Belle de Mai (Marseille) et le Flon (Lausanne) », Géocarrefour, vol. 81, nº 2, 2006, p. 159-166.

Bachimon, Philippe. "Paradoxales friches urbaines ", Linformation géographique, vol. 78, $\mathrm{n}^{\circ}$ 2, 2014, p. 42-61.

Bailly, Émeline, "Franges intra-urbaines à l'épreuve des projets de paysage ", Projets de Paysage. 2016, http://www.projetsdepaysage.fr/fr/_ franges_intra_urbaines_1_preuve_des_projets_de_paysage, site consulté le 2 septembre 2018.

Bambra, Clare et al., " Healthy Land? An Examination of the Area-Level Association between Brownfield Land and Morbidity and Mortality in England ", Environment and Planning A: Economy and Space, vol. $46, \mathrm{n}^{\circ}$ 2, 2014, p. 433-454.

Bambra, Clare et al., "This Divided Land: An Examination of Regional Inequalities in Exposure to Brownfield Land and the Association with Morbidity and Mortality in England ", Health and Place, $\mathrm{n}^{\circ} 34,2015$, 257-69.

Beames, Alistair et al., "Amenity Proximity Analysis for Sustainable Brownfield Redevelopment Planning», Landscape and Urban Planning, $\mathrm{n}^{\circ}$ 171, 2017, p. 68-79.

Bonthoux, Sébastien, Marion Brun, Francesca Di Pietro, Sabine Greulich et Sabine Bouché-Pillon, «How Can Wastelands Promote Biodiversity in Cities? A Review ", Landscape and Urban Planning, $\mathrm{n}^{\circ}$ 132, 2014, p. 79-88.

Boutefeu, Emmanuel, La demande sociale de nature en ville, enquête auprès des habitants de l'agglomération lyonnaise, Paris, PUCA/CERTU, 2005.

Brun, Marion, Sébastien Bonthoux, Sabine Greulich et Francesca Di Pietro, «Les services de support de diversité floristique rendus par les délaissés urbains ", Environnement Urbain / Urban Environment, n ${ }^{\circ}$ 11, 2017, https://journals.openedition.org/eue/1500, consulté le consulté le 12 juin 2012. 
Brun, Marion, Francesca Di Pietro et Sébastien Bonthoux, "Residents' Perceptions and Valuations of Urban Wastelands Are Influenced by Vegetation Structure ", Urban Forestry and Urban Greening, n ${ }^{\circ} 29$, 2018, p. 393-403.

Brun, Marion, Lucy Vaseux, Denis Martouzet et Francesca Di Pietro, "Usages et représentations des délaissés urbains, supports de services écosystémiques culturels en ville ", Environnement Urbain / Urban Environment, $\mathrm{n}^{\circ}$ 11, 2017, http://journals.openedition.org/eue/1906, consulté le 12 juin 2012.

Calenge, Christian, "L'idéologie verte et la rhétorique paysagère ", Communications, $\mathrm{n}^{\circ} 74,2003$, p. 33-47.

Chrysochoou, Maria et al., "A GIS and Indexing Scheme to Screen Brownfields for Area-Wide Redevelopment Planning ", Landscape and Urban Planning, $\mathrm{n}^{\circ}$ 105, 2012, p. 187-98.

Clément, Gilles, Manifeste du tiers paysage, Montreuil, Sujet/Objet éditions, 2007.

Darly, Ségolène, Pauline Marty et Johan Milian, "La "nature en ville" à l'épreuve de la requalification des banlieues. Le cas de PlaineCommune ", 20 novembre 2013, p. 1-6, https://www.metropolitiques. eu/La-nature-en-ville-a-l-epreuve-de.html, consulté le 12 février 2018.

De Sousa, Christopher A., "Turning Brownfields into Green Space in the City of Toronto ", Landscape and Urban Planning, n ${ }^{\circ}$ 62, 2003, p. 181-198.

Doick, Kieron J., Gloria Sellers, Vanesa Castán Broto et T. Silverthorne, "Understanding Success in the Context of Brownfield Greening Projects: The Requirement for Outcome Evaluation in Urban Greenspace Success Assessment ", Urban Forestry and Urban Greening, vol. 8, n 3, 2009, p. 163-78.

Drake, Luke et Laura J. Lawson, "Validating Verdancy or Vacancy? The Relationship of Community Gardens and Vacant Lands in the U.S. ", Cities, $n^{\circ}$ 40, 2014, p. 133-42.

Émelianoff, Cyria, «Urbanisme durable? ». Écologie and politique, vol. 29, $n^{\circ} 2,2004$, p. 13-19.

Fallery, Bernard et Florence Rodhain, «Quatre approches pour l'analyse de données textuelles ", $\mathrm{XVI}^{e}$ Conférence de l'Association Internationale de Management Stratégique, Montréal, Canada, 2007, p. 1-16, https://hal.archives-ouvertes.fr/hal-00821448, consulté le 16 août 2018.

Fischer, Leonie K., Moritz Von der Lippe et Ingo Kowarik « Urban Land Use Types Contribute to Grassland Conservation: The Example of 
Berlin ", Urban Forestry and Urban Greening, vol. 12, n 3, 2013, p. 263-272.

Foo, Katherine, Deborah Martin, Clara Wool et Colin Polsky, "The Production of Urban Vacant Land: Relational Placemaking in Boston, MA Neighborhoods ", Cities, no 40, 2014, p. 175-82.

Foster, Jennifer, "Hiding in Plain View: Vacancy and Prospect in Paris' Petite Ceinture ", Cities, no 40, 2014, p. 124-132.

Ghiglione, Rodolphe et Benjamin Matalon, Les enquêtes sociologiques. Théories et pratique, Paris, Armand Colin, coll. "U : sociologie », 1998. Gobster, Paul H. et Lynne M. Westphal, «The Human Dimensions of Urban Greenways: Planning for Recreation and Related Experiences ", Landscape and Urban Planning, vol. 68, ${ }^{\circ \mathrm{os}} 2-3,2004$, p. 147-165.

Grésillon, Lucile, Sentir Paris. Bien-être et matérialité des lieux, Versailles, Quae, coll. «Indisciplines », 2010.

Haaland, Christine et Cecil Konijnendijk van den Bosch, «Challenges and Strategies for Urban Green-Space Planning in Cities Undergoing Densification: A Review ", Urban Forestry and Urban Greening, vol. 14, $\mathrm{n}^{\circ}$ 4, 2015, p. 760-771.

Harrison, Carolyn et Gail Davies, « Conserving Biodiversity That Matters: Practitioners' Perspectives on Brownfield Development and Urban Nature Conservation in London ", Journal of Environmental Management, vol. 65, $\mathrm{n}^{\circ}$ 1, 2002, p. 95-108.

Herbst, Harriet et Volkmar Herbst, «The Development of an Evaluation Method Using a Geographic Information System to Determine the Importance of Wasteland Sites as Urban Wildlife Areas ", Landscape and Urban Planning, vol. 77, ${ }^{\text {os }} 1-2,2006$, p. 178-195.

Hofmann, Mathias, Janneke R. Westermann, Ingo Kowarik et Elke Van der Meer, « Perceptions of Parks and Urban Derelict Land by Landscape Planners and Residents ", Urban Forestry and Urban Greening, vol. 11, no 3, 2012, p. 303-312.

Kim, Minseo, Christoph Rupprecht et Katsunori Furuya, "Residents' Perception of Informal Green Space. A Case Study of Ichikawa City, Japan ", Land, vol. 7, no 3, 2018, https://www.mdpi.com/2073445X/7/3/102, consulté le 15 juin 2016.

Kobayashi, Takeshi et Shinji Ikaruga, " Occurrence Factors of Large Vacant Lots in Central Districts and Their Utilization by Local Governments in Japan ", Frontiers of Architectural Research, vol. 5, n' 4, 2016, p. 393-402.

Kowarik, Ingo et Andreas Langer, "Natur-Park Südgelände: Linking Conservation and Recreation in an Abandoned Railyard in Berlin ", 
dans Ingo Kowarik and Stefan Körner (dir.), Wild Urban Woodlands: New Perspectives for Urban Forestry, Berlin, Springer-Verlag, 2005, p. 287-299.

Kowarik, Ingo, "The "Green Belt Berlin”: Establishing a Greenway Where the Berlin Wall Once Stood by Integrating Ecological, Social and Cultural Approaches ", Landscape and Urban Planning, ${ }^{\circ}$ 184, 2019, p. 12-22.

Krekel, Christian, Jens Kolbe et Henry Wüstemann, "The Greener, The Happier? The Effects of Urban Green and Abandoned Areas on Residential Well-Being ", Ecological Economics, n ${ }^{\circ}$ 121, 2016, p. 117-27.

Kremer, Peleg, Zoe Hamstead et Timon McPhearson, «A Social-Ecological Assessment of Vacant Lots in New York City ", Landscape and Urban Planning, $\mathrm{n}^{\circ} 120,2013$, p. 218-33.

Jensen, Ebba Lisberg et Pernilla Ouis, "Contested Construction of Nature for City Fringe Outdoor Recreation in Southern Sweden. The Arrie Case ", Urban Forestry and Urban Greening, vol. 7, n 3, 2008, p. 171-82.

Lafortezza, Raffaele, Robert C. Corry, Giovanni Sanesi et Robert D. Brown, «Visual Preference and Ecological Assessments for Designed Alternative Brownfield Rehabilitations ", Journal of Environmental Management, vol. 89, n 3, p. 257-69.

Lévesque, Luc, "Montréal, l'informe urbanité des terrains vagues pour une gestion créatrice du mobilier urbain ", Les Annales de la recherche urbaine, $\mathrm{n}^{\circ} 85,1999$, p. 47-57.

Lemoine, Guillaume, "Usages temporaires des friches urbaines de l'Établissement Public Foncier Nord-Pas-de-Calais : une contribution aux villes durables? ", Techniques, sciences, méthodes, $\mathrm{n}^{\circ}$ 3, 2017, p. 1-8.

Litt, Jill et Thomas A. Burke, "Uncovering the Historic Environmental Hazards of Urban Brownfields ", Journal of Urban Health, vol. 79, $\mathrm{n}^{\circ}$ 4, 2002, p. 464-81.

Lizet, Bernadette, "Du terrain vague à la friche paysagée ", Ethnologie française, $\mathrm{n}^{\circ} 4,2010$, p. 597-608.

Lizet, Bernadette, «Naturalistes, herbes folles et terrains vagues », Ethnologie française, vol. 19, n 3, 1989, p. 253-272.

Mathey, Juliane, Thomas Arndt, Juliane Banse et Dieter Rink, "Public Perception of Spontaneous Vegetation on Brownfields in Urban Areas-Results from Surveys in Dresden and Leipzig (Germany)", Urban Forestry and Urban Greening, n' 29, 2018, p. 384-392. 
Menozzi, Marie-Jo, " "Mauvaises herbes”, qualité de l'eau et entretien des espaces ", Natures, sciences, sociétés, vol. 15, n 2, 2007, p. 144-153.

Miller, James R. et Richard J. Hobbs, "Conservation Where People Live and Work ", Conservation Biology, vol. 16, n 2, 2002, p. 330-337.

Moser, Gabriel et Karine Weiss, Espaces de vie. Aspects de la relation hommeenvironnement, Paris, Armand Colin, 2003.

Orduña-Giró, Paula et Sébastien Jacquot, "La production participative d'espaces publics temporaires en temps de crise. Le projet "Pla Buits" à Barcelone», Métropolitiques, 7 novembre 2014, https://www.metropolitiques.eu/La-production-participative-d.html, consulté le 31 décembre 2017.

Pizzol, Lisa et al., "Timbre Brownfield Prioritization Tool to Support Effective Brownfield Regeneration ", Journal of Environmental Management, 2016, $\mathrm{n}^{\circ}$ 166, p. 178-92.

Plottu, Béatrice et Majorie Tendero, "Acceptabilité des choix d'aménagement des friches urbaines polluées ", Revue d'économie régionale et urbaine, $\mathrm{n}^{\circ} 2,2017,335-353$.

Rall, Emily Lorance et Dagmar Haase, "Creative Intervention in a Dynamic City: A Sustainability Assessment of an Interim Use Strategy for Brownfields in Leipzig, Germany ", Landscape and Urban Planning, vol. 100, no 3, 2011, p. 189-201.

Reygrobellet, Bernard, La nature dans la ville. Biodiversité et urbanisme, Paris, La Documentation française, 2007.

Riley, Christopher B., Kayla I. Perry, Kerry Ard et Mary M. Gardiner, "Asset or Liability? Ecological and Sociological Tradeoffs of Urban Spontaneous Vegetation on Vacant Land in Shrinking Cities», Sustainability, vol. 10, n ${ }^{\circ}$ 7, 2018, p. 1-19.

Rink, Dieter et Harriet Herbst, «From Wasteland to Wilderness. Aspects of a New Form of Urban Nature ", dans Matthias Richter et Ulrike Weiland (dir.), Applied Urban Ecology: A Global Framework, Chichester, West Sussex (UK), Wiley-Blackwell, 2012, p. 82-92.

Rupprecht Christoph D. D. et Jason A. Byrne, "Informal Urban Greenspace: A Typology and Trilingual Systematic Review of Its Role for Urban Residents and Trends in the Literature », Urban Forestry and Urban Greening, vol. 13, n 4, 2014, p. 597-611.

Rupprecht, Christoph D. D. et Jason A. Byrne, «Informal Urban GreenSpace: Comparison of Quantity and Characteristics in Brisbane, Australia and Sapporo, Japan», PLoS ONE, vol. 9, n 6, 2014, https:// journals.plos.org/plosone/article?id=10.1371/journal.pone.0099784, consulté le 12 juin 2017. 
Rupprecht Christoph D. D., Jason A. Byrne, Hirofumi Ueda et Alex Y. Lo, " "It's Real, Not Fake Like a Park": Residents' Perception and Use of Informal Urban Green-Space in Brisbane, Australia and Sapporo, Japan ». Landscape and Urban Planning, ${ }^{\circ}$ 143, 2015, p. 205-218.

Sénécal, Gilles et Diane Saint-Laurent, "Espaces libres et enjeux écologiques : deux récits du développement urbain à Montréal », Recherches sociographiques, vol. $40, \mathrm{n}^{\circ} 1,1999$, p. 33-54.

Schwartz, Assaf et al., "Outstanding Challenges for Urban Conservation Research and Action ", Global environmental change, vol. 28, $\mathrm{n}^{\circ} 1$, 2014, p. 39-49.

Thomas, Michael R., "A GIS-Based Decision Support System for Brownfield Redevelopment ", Landscape and Urban Planning, vol. 58, $\mathrm{n}^{\circ}$ 1, 2002, p. 7-23.

Threlfall, Caragh G. et Dave Kendal, «The Distinct Ecological and Social Roles That Wild Spaces Play in Urban Ecosystems ", Urban Forestry and Urban Greening, $\mathrm{n}^{\circ}$ 29, 2017, p. 1-9.

Tzoulas Konstantinos et Philip James, "Peoples' Use of, and Concerns about, Green Space Networks: A Case Study of Birchwood, Warrington New Town, UK », Urban Forestry and Urban Greening, vol. 9, $\mathrm{n}^{\circ} 2$, 2010, p. 121-128.

Zaninetti, Jean-Marc et Dominique Andrieu, «L'étalement résidentiel des métropoles intermédiaires de la région Centre Val de Loire ", Mappemonde, $\mathrm{n}^{\circ} 124,2018$, p. 1-21. 